\title{
Efeitos dos Programas de Transferência de Renda sobre a Oferta de Trabalho Não Agrícola na Área Rural da Região Nordeste
}

\author{
José Antonio Nunes ${ }^{1}$ e Jorge Luiz Mariano²
}

\begin{abstract}
Resumo: O objetivo do estudoéinvestigar se os programas sociais e de transferência de renda afetam as decisões dos filhos e dos pais de participação e de alocação de horas de trabalho em atividades não agrícolas. A hipótese testada pressupõe que o acesso aos programas sociais e de transferência de renda contribui para desestimular a participação nessas atividades. A metodologia consiste no uso dos modelos de Heckman (1979) e de double hurdle, de Cragg (1971), que associam a decisão de participação no mercado de trabalho com a decisão de alocação de horas de trabalho. A base de dados utilizada foi a da Pesquisa Nacional por Amostragem de Domicílio (PNAD), do ano de 2006. Os resultados mostraram que os programas sociais têm influenciado, negativamente, a participação de pais e filhos na oferta de trabalho não agrícola. Observou-se, ainda, que o efeito do programa Bolsa Família de reduzir as chances de participação dos jovens em atividades não agrícolas pode estar relacionado à condicionalidade da frequência escolar.
\end{abstract}

Palavras-chaves: Atividades não agrícolas, transferência de renda, oferta de trabalho.

Abstract: The objective of this study is to analyze the effect of social programs and cash transfer on the labor supply of non-farm family members, in poverty conditions, in rural areas of the Northeastern region, in Brazil. The hypothesis indicates that access to social programs and cash transfers may contribute to discourage the rural workers, in poverty, in their decision to participate and offer hours of work in non-agricultural activities. The methodology consisted on the use of models of Heckman (1979) and double hurdle, of Cragg (1971), which seek to associate the decision to participate in the labor market with the decision to allocate working hours. The database used was the National Survey by Household Sampling (PNAD), of 2006. Furthermore, the estimates for the parent and children showed that the programs have impacted negatively on the participation in non-agricultural activities. It has also been observed that the effects of Bolsa Familia

1. Mestre em Economia. Professor da Universidade Potiguar. E-mail: economist.30@hotmail.com

2. Doutor em Economia. Professor da Universidade Federal do Rio Grande do Norte. E-mail: jdal@ufrnet.br 
programs reduce the chance of teenagers' participation in the non-agricultural activities, given that it is related to conditionality of school frequency.

Key-words: Non-agricultural activities, cash transfer, labor supply.

Classificação JEL: I3, Q1, J2.

DOI: http://dx.doi.org/10.1590/1234-56781806-9479005301004

\section{Introdução}

Ao longo das últimas décadas, vários estudos evidenciaram a importância das atividades não agrícolas nos espaços rurais. Dentre esses estudos, os de Klein (1992), Del Grossi e Graziano da Silva (2000) e Davis (2004) destacam a participação dessas atividades na diversificação das fontes de renda e do emprego nas áreas rurais. Reardon et al. (2006) admitem que as famílias rurais se engajam nas atividades não agrícolas incentivadas pelos maiores retornos ou menores riscos em relação às atividades estritamente agrícolas. Além dos períodos de entressafra, choques adversos sobre a produção rural - por exemplo: secas prolongadas, queda brusca de preços agrícolas, degradação ambiental - permitem que as atividades não agrícolas se tornem importante alternativa de sobrevivência para as famílias do meio rural.

Del Grossi et al. (2001), estudando o diferencial de rendimentos entre as ocupações rurais, mostraram que, entre as pessoas ocupadas no meio rural, a maior renda média era a dos trabalhadores por conta própria não agrícolas. No recorte regional, alguns estados da região Nordeste têm uma característica distinta das dos demais quando se trata das atividades não agrícolas. Gomes da Silva (2000), em pesquisa realizada na região, constatou que, no Rio Grande do Norte, a população economicamente ativa ocu- pada, no meio rural, em atividades não agrícolas, superava o número de pessoas ocupadas em atividades agrícolas. Na região da Zona da Mata, em Pernambuco e Alagoas, onde a agroindústria da cana-de-açúcar se destaca como uma atividade econômica predominante, as maiores ocupações rurais não agrícolas estavam nos setores da indústria de transformação e de serviços. Del Grossi e Graziano da Silva (2000) mostraram que as atividades estritamente agrícolas, ao longo do tempo, vêm reduzindo sua participação na alocação de força de trabalho das famílias rurais, principalmente na região Nordeste.

Davis (2004) ressalta que a disseminação das atividades não agrícolas nas áreas rurais tem recebido grande destaque na literatura, uma vez que elas são capazes de gerar efeitos significativos sobre o nível de renda e de emprego no campo e, em muitos casos, são a única forma de sobrevivência das famílias rurais. A importância dessas atividades se faz presente principalmente nas áreas rurais em que os índices de pobreza são mais fortes.

Em relação à pobreza, Ferreira e Lanjouw (2001), estudando a região Nordeste do Brasil, observaram que a incidência da pobreza é bem maior nas áreas rurais do que nas áreas urbanas. Constataram que $48,8 \%$ das famílias rurais eram pobres, enquanto nas áreas urbanas esse percentual era de apenas $30 \%$ da população.

De acordo com Neder e Silva (2004), as políticas de combate à pobreza devem considerar a 
importância da renda não agrícola na composição da renda das famílias pobres. Os autores apontam que as atividades não agrícolas podem servir como estratégia para reduzir o número de famílias que estão abaixo da linha da pobreza e em condições de indigência.

A criação do programa Bolsa Família e a ampliação dos programas sociais - como o Benefício de Prestação Continuada (BPC) e as aposentadorias - permitiram maior focalização das políticas públicas nas camadas mais pobres da população, com efeitos significativos na redução da pobreza rural e urbana em todo o País e, de forma particular, na região Nordeste, onde os indicadores da pobreza são mais perversos. Hoffmann (2006) destacou a importância desses programas na redução da desigualdade de renda e da pobreza, na região Nordeste, entre os anos de 2002 e 2004.

Paralelamente aos estudos de pesquisadores que analisaram os efeitos dos programas sociais e de transferência de renda no alívio da pobreza, outros procuraram analisar a influência desses programas sobre a decisão do público beneficiado de participar do mercado de trabalho. Mathse e Young (2004), estudando as decisões de oferta de trabalho não agrícola no Zimbábue, perceberam que as transferências de renda causaram um efeito negativo sobre a probabilidade de as famílias ofertarem horas de trabalho não agrícola. Ferro e Nicolela (2007) mostraram que, tanto nas áreas urbanas quanto nas rurais, o programa de transferência condicional de renda Bolsa Família reduziu a probabilidade de meninos e meninas trabalharem; porém, não afetou a decisão destes de alocar horas de trabalho. Os autores ainda constataram que a participação dos pais nas atividades não agrícolas não foi afetada pelo acesso ao programa, mas, que as horas de trabalho alocadas mudaram devido ao recebimento do Bolsa Família.

Pergunta-se, então: se os programas de transferência de renda ajudaram a reduzir a pobreza nas áreas rurais do Nordeste, eles teriam afetado negativamente as decisões sobre participação e de alocação de horas de trabalho nas ativida- des não agrícolas? Em que sentido os programas sociais e de transferência condicional de renda influenciam pais e filhos na decisão sobre participação e sobre alocação de horas de trabalho em atividades não agrícolas na área rural da região Nordeste?

Na tentativa de responder a essas questões, este estudo tem como objetivo investigar se os programas sociais e de transferência condicional de renda afetam a decisão dos filhos e dos pais pobres (chefes de família) de alocar horas de trabalho em atividades não agrícolas na região rural do Nordeste.

A estratégia metodológica do estudo consiste na utilização de dois modelos para analisar os efeitos dos programas de transferência de renda sobre a decisão de participação e alocação de horas de trabalho das famílias beneficiadas. Um deles é o modelo de Heckman (1979), que permite observar, em duas etapas, a decisão de ofertar horas de trabalho: a primeira é relativa à participação e a segunda é relativa à decisão de alocação de horas de trabalho (condicionada à primeira). Também se utiliza o modelo de Cragg (1971), conhecido como double hurdle, que permite a estimação dos determinantes da participação e da alocação de horas de trabalho em atividades não agrícolas, por meio de decisões distintas, porém sequenciais. Esse modelo também foi utilizado no trabalho de Mesquita et al. (2010), para analisar a oferta de trabalho não agrícola no meio rural na região Nordeste. Entretanto, este estudo se diferencia do trabalho de Mesquita et al. (2010) por incluir os programas sociais na análise sobre a decisão de participação e de oferta de horas de trabalho em atividades não agrícolas por parte dos chefes e dos jovens de famílias pobres do meio rural do Nordeste.

A delimitação da área do estudo justifica-se pela constatação, em estudos, como o de Rocha (2011), de que a maioria dos pobres do Brasil está na região Nordeste, predominantemente no meio rural, que vem apresentando, ao longo dos anos, uma diversidade de atividades com características urbanas e que servem como alternativas de ocupação e renda para as pessoas que vivem 
nessa região. Considerou-se, ainda, na delimitação da área do estudo, a importância dos programas sociais na composição da renda e na redução da pobreza dessa região.

Este trabalho está organizado em quatro seções, além desta introdução. Na segunda, apresentam-se, de forma sumária, os principais programas sociais e de transferência de renda e também alguns estudos que procuraram observar o efeito dos programas de transferência condicional de renda sobre a decisão de trabalhar e a de alocar horas de trabalho das famílias assistidas. $\mathrm{Na}$ terceira seção, apresentam-se a base de dados e os procedimentos metodológicos para estimação dos modelos de Heckman (1979) e de Cragg (1971); na quarta, os resultados; e, na última, são elencadas as principais constatações do estudo e as considerações finais.

\section{Programas de transferências de renda e oferta de trabalho}

Com o objetivo de reduzir os níveis de pobreza no Brasil, o governo federal vem implantando um conjunto de políticas de transferência direta de renda, procurando focalizar as populações de baixa renda, tanto nas áreas urbanas como nas áreas rurais do País.

O marco institucional dessas políticas é a Constituição de 1988 e a Lei Orgânica da Assistência Social (LOAS), de 1993, que trouxe rumo completamente novo ao tratamento da assistência social no Brasil. Desde então, diversos programas de transferência direta de renda foram sendo instituídos, focalizando a população em situação de pobreza ou que não dispõe dos recursos básicos para sua sobrevivência. Destes, o programa Bolsa Família (PBF), o Benefício de Prestação Continuada (BPC) e o Programa de Erradicação do Trabalho Infantil (Peti) se destacaram como os principais programas de transferência de renda do governo federal e foram considerados na Pesquisa Nacional por Amostragem de Domicilio do ano de 2006.

Criado em 2003 pelo governo federal, o Bolsa Família unificou programas sociais existentes na época, como o Bolsa Alimentação, o Cartão Alimentação, o Bolsa Escola e o Auxílio Gás. O programa tornou-se a principal política de transferência direta de renda do governo federal para as famílias que estão em situação de pobreza ou de extrema pobreza. Para receber os benefícios, as famílias devem inscrever-se no Cadrasto Único de Programas Sociais (CadÚnico). São classificadas como pobres aquelas famílias que têm renda per capita inferior a $\mathrm{R} \$ 154,00$ e, como extremamente pobres aquelas que têm renda per capita interior a $\mathrm{R} \$ 77,00$.

As famílias selecionadas pelo Ministério de Desenvolvimento Social e Combate à Fome (MDS) passam a receber os benefícios do programa, assumindo o compromisso de manter as crianças e os jovens de 6 a 17 anos na escola, acompanhar o cartão de vacinação e desenvolvimento de menores de sete anos, e as gestantes e nutrizes o de fazer o acompanhamento de sua saúde e da saúde da criança. As famílias beneficiadas - em que há adolescentes com até 15 anos, retirados do trabalho infantil pelo Programa de Erradicação do Trabalho Infantil (Peti) - devem obter frequência de $85 \%$ dos serviços de convivência e fortalecimento de vínculos.

Conforme o MDS, o Bolsa Família visa alcançar três eixos: o primeiro refere-se à transferência imediata de renda, com o objetivo de aliviar as famílias que estão na condição de pobreza e na de extrema pobreza; o segundo concentra-se nas condições requeridas para recebimento e manutenção dos benefícios, que fortalecem o acesso aos direitos sociais básicos das crianças e dos jovens nas áreas de educação, saúde e assistência social; e o terceiro eixo possibilita que as crianças e os adolescentes em situação de vulnerabilidade não retornem ao trabalho infantil.

Em relação à sua cobertura, ao longo dos anos, o programa ganhou impulso em termos de famílias beneficiadas e valores repassados. Dados da Tabela 1 mostram que, desde o início desse programa, o Nordeste é a região com maior número de famílias beneficiadas (51\% de 13 milhões de famílias em todo Brasil, em 2011) e com os maiores valores repassados $(51,4 \%$ dos 16 bilhões 
Tabela 1. Bolsa Família - número de famílias beneficiadas, valores liberados - Brasil e regiões, 2004 e 2011

\begin{tabular}{|c|c|c|c|c|c|c|c|c|}
\hline \multirow[b]{2}{*}{ Regiões } & \multicolumn{4}{|c|}{2004} & \multicolumn{4}{|c|}{2011} \\
\hline & $\begin{array}{l}\text { Famílias } \\
\text { (em mil) }\end{array}$ & $\%$ & $\begin{array}{c}\text { Valores } \\
\text { (em mil) }\end{array}$ & $\%$ & $\begin{array}{l}\text { Famílias } \\
\text { (em mil) }\end{array}$ & $\%$ & $\begin{array}{c}\text { Valores } \\
\text { (em mil) }\end{array}$ & $\%$ \\
\hline Centro-Oeste & 292 & 4.4 & 17.540 & 4.0 & 717 & 5.4 & 82.736 & 5.2 \\
\hline Norte & 527 & 8.0 & 37.758 & 8.6 & 1.476 & 11.1 & 198.389 & 12.4 \\
\hline Nordeste & 3.320 & 50.5 & 234.533 & 53.3 & 6.825 & 51.1 & 823.668 & 51.4 \\
\hline Sul & 700 & 10.7 & 42.069 & 9.6 & 1.035 & 7.8 & 118.127 & 7.4 \\
\hline Sudeste & 1730 & 26.3 & 107.969 & 24.5 & 3.296 & 24.7 & 379.157 & 23.7 \\
\hline Brasil & 6.571 & 100.0 & 439.870 & 100.0 & 13.352 & 100.0 & 16.02.079 & 100.0 \\
\hline
\end{tabular}

Fonte: Brasil, Ministério do Desenvolvimento Social, 2013. Elaboração dos autores.

repassados em 2011). Essa distribuição mostra a forma distinta da cobertura do programa entre as regiões. Muito provavelmente, os critérios de cobertura estão relacionados aos maiores índices de proporção de pobres e de pobreza extrema na região Nordeste, em relação às outras regiões.

$\mathrm{O}$ programa BPC se destina à transferência de um salário mínimo para pessoas com mais de 65 anos de idade e deficientes que comprovadamente estejam incapacitadas para a vida independente e para o trabalho. Observa-se, na Tabela 2, que a região Nordeste também se destaca como a que concentra o maior número de beneficiários e de valores repassados para a modalidade de pessoas idosas.

Para Graziano da Silva et al. (2002), as famílias rurais mais pobres têm grande dependência da transferência de renda sob a forma de aposentadoria e de transferências sociais. Nas áreas rurais do Nordeste, as transferências sociais são componentes importantes da renda dos domicílios rurais, sobretudo pelo fato de que a região ainda apresenta os mais elevados índices de pobreza rural (FERREIRA e LANJOUW, 2001).

Conforme Soares et al. (2007), as evidências dos efeitos negativos dos programas de transferência de renda sobre a oferta de trabalho não parecem ter consistência empírica e, quando esses são constatados, eles acontecem em atividades em que as condições de trabalho são mais penosas e insalubres. Os autores mostraram que as pessoas que recebem transferência de renda de programas sociais trabalham igualmente às não beneficiadas ou até mais que estas.

Considera-se que o valor dos benefícios e algumas condições para o recebimento das transferências não desestimulam a oferta de trabalho das famílias beneficiadas. No primeiro caso, os benefícios não teriam valores altos o suficiente para que os membros adultos optassem por reduzir suas horas de trabalho; no segundo caso, os condicionantes representariam uma porta de entrada para o mercado de trabalho, uma vez que rompem alguns obstáculos que impediam o

Tabela 2. Benefício de Prestação Continuada - número de beneficiários, valores liberados Brasil e regiões, 2004 e 2011

\begin{tabular}{l|cccc|cccc}
\hline \multicolumn{1}{c|}{ Regiões } & \multicolumn{4}{c|}{$\mathbf{2 0 0 4}$} & \multicolumn{2}{c}{$\mathbf{2 0 1 1}$} \\
\hline Sudeste & $\begin{array}{c}\text { Beneficiários } \\
\text { (em mil) }\end{array}$ & $\%$ & $\begin{array}{c}\text { Valores (em } \\
\text { mil) }\end{array}$ & $\%$ & $\begin{array}{c}\text { Beneficiários } \\
\text { (em mil) }\end{array}$ & $\%$ & $\begin{array}{c}\text { Valores (em } \\
\text { mil) }\end{array}$ & $\begin{array}{c}\text { \% } \\
\text { Sul }\end{array}$ \\
Nordeste & 13 & 33.2 & 1.563 & 33.1 & 162 & 9.6 & 88.459 & 9.6 \\
Norte & 16 & 8.0 & 376 & 8.0 & 161 & 9.6 & 88.109 & 9.6 \\
Centro-Oeste & 3 & 38.7 & 1.827 & 38.7 & 539 & 32.0 & 293.726 & 32.0 \\
Brasil & 4 & 8.9 & 421 & 8.9 & 165 & 9.8 & 90.302 & 9.8 \\
\hline
\end{tabular}

Fonte: Brasil, Ministério do Desenvolvimento Social, 2013. Elaboração dos autores. 
acesso a melhores trabalhos, principalmente com a elevação do nível educacional.

Alguns estudos procuraram verificar o impacto das transferências de renda na oferta de trabalho, chegando a conclusões distintas. Teixeira (2008) analisou o efeito do programa Bolsa Família na probabilidade de trabalhar de homens e mulheres, através de uma estimação em dois estágios. Ele argumenta que, embora o efeito na redução de horas de trabalho tenha se mostrado significativo, não foi de magnitude que justifique afirmar que o programa reduz a oferta de trabalho. O autor mostrou, ainda, que essa redução ocorre com frequência nos trabalhos mais precários.

Nessa mesma linha de raciocínio, Tavares (2008) estudou o impacto do PBF na oferta de trabalho das mães. O autor utilizou o procedimento econométrico de propensity-score matching, que consiste em selecionar, dentro da amostra, um grupo de controle, para comparar com um grupo de tratamento. Os resultados não confirmaram a hipótese de que as transferências possam ter um impacto negativo sobre a oferta de trabalho: ele observou que as mães beneficiadas apresentaram maior probabilidade tanto de participação no mercado de trabalho como também de ofertar jornadas maiores de trabalho do que as mães não beneficiárias.

Ferro e Nicollela (2007) utilizaram o método do propensity-score matching, com o modelo Probit, na decisão de participação e o modelo de Heckman two steps na determinação das horas alocadas. Os autores observaram que o recebimento de renda dos programas sociais não alterava a probabilidade de participação dos membros adultos, mas, sim, provocava uma mudança na quantidade de horas trabalhadas. Eles encontraram um efeito negativo dos programas sociais sobre a participação dos homens, tanto nas áreas urbanas como nas rurais, bem como sobre a das mulheres das áreas urbanas. Os autores apontaram que isso se daria pelo fato de os pais precisarem de mais tempo para cuidar dos filhos em decorrência das exigências do programa.

Fogel e Barros (2010) investigaram os impactos dos programas de transferência de renda na oferta de trabalho de homens e mulheres nos municípios brasileiros. Os principais resultados mostraram um efeito negativo para as mulheres e um não significativo para os homens. Os autores concluíram que os resultados eram compatíveis com a premissa de que os programas de transferência de renda não afetam a decisão de oferta de trabalho dos beneficiários homens.

Outras pesquisas acerca da influência dos programas sociais de transferência de renda evidenciaram impacto positivo no sentido da redução da oferta de trabalho infantil. Ferro e Kassouf (2005) estudaram os efeitos do programa Bolsa Escola, utilizando um modelo de estimação Probit para a participação no mercado de trabalho e o de mínimos quadrados ordinários para a alocação de horas trabalhadas. Chegaram à conclusão de que o programa produziu um impacto positivo na redução do trabalho infantil, diminuindo a probabilidade de participação das crianças.

Em síntese, embora em alguns estudos se constate a presença do efeito negativo das transferências na oferta de trabalho, a maioria dos pesquisadores entende que esse efeito não tem magnitude suficiente para que se afirme, consistentemente, que os programas de transferência de renda operam no sentido de reduzir a oferta de trabalho, mesmo que nos trabalhos mais precários essa afirmação possa fazer sentido, como sugerem alguns autores.

\section{Metodologia}

Para analisar a relação entre oferta de trabalho e transferência de renda, adotou-se o modelo de Cragg (1971) e o de Heckman (1979). O primeiro será utilizado para estimar as equações de decisões de participação e de alocação de horas de trabalho dos pais e dos filhos, e o segundo modelo será utilizado na equação dos pais para verificar a consistência do modelo de duble hurdle de Cragg. Conforme Wodjao (2007) e Mesquita et al. (2010), o modelo de duble hurdle possui a vantagem, quando comparado com o modelo de Heckman, de permitir incluir, na equação de alocação de 
horas trabalhadas, os indivíduos que decidiram participar em atividades não agrícolas, mas, por algum motivo, não ofertaram horas de trabalho. Essa estratégia de comparação de modelos também foi adotada por Detre et al. (2010), Aristei e Pieroni (2011) e Setsoffia et al. (2013).

Heckman (1979) formulou um modelo que aborda o problema associado com observações zero geradas pela decisão de não participação, argumentando que estimativas feitas em subamostras selecionadas podem levar a erros amostrais. O modelo de Heckman procura resolver esse problema através de uma estimação em dois estágios. Inicialmente, realiza-se uma estimação pelo modelo de regressão Probit em toda a amostra e, em seguida, faz-se uma estimação censurada na subamostra selecionada. Assim, na primeira decisão, estima-se a probabilidade de participação e, na segunda, estima-se o nível de horas de trabalho, condicionada à primeira.

Conforme Wodjao (2007), o modelo de Heckman pode ser enunciado em duas etapas, assim representadas:

Decisão de participação:

$$
\begin{aligned}
& \mathrm{d}_{\mathrm{i}}^{*}=\mathrm{X}_{\mathrm{ii}}^{\prime} \mathrm{b}_{1}+\mathrm{m}_{\mathrm{i}}, \quad \mathrm{m}_{\mathrm{i}} \sim \mathrm{N}(0,1) \\
& \mathrm{d}_{\mathrm{i}}= \begin{cases}1 & \text { se } \mathrm{d}_{\mathrm{i}}^{*}>0 \\
0 & \text { se } \mathrm{d}_{\mathrm{i}}^{*} \leq 0\end{cases}
\end{aligned}
$$

Nível de participação:

Horas alocadas $\mathrm{t}_{\mathrm{i}}^{*}=\mathrm{X}_{2 \mathrm{i}}^{\prime} \mathrm{b}_{2}+\mathrm{v}_{\mathrm{i},} \quad \mathrm{v}_{\mathrm{i}} \sim \mathrm{N}\left(0, \mathrm{~s}^{2}\right)$

$$
t_{i}=\left\{\begin{array}{lll}
t_{i}^{*} & \text { se } d_{i}=1 \\
0 & \text { se } d_{i}=0
\end{array}\right.
$$

Nessas expressões, $X_{1 i}^{\prime}$ e $X_{2 i}^{\prime}$ são vetores de variáveis explicativas nos dois estágios de decisão. Assume-se que ambas as variáveis não são correlacionadas com os respectivos erros: $\mu_{\mathrm{i}}$ e $\mathrm{v}_{\mathrm{i}}$; $\beta_{\mathrm{i}}$ e $\beta_{2}$ são os vetores dos parâmetros; $\mathrm{d}_{\mathrm{i}}^{*}$ é uma variável binária que representa a decisão individual de participação nas atividades não agrícolas.

Na equação de alocação de horas de trabalho, $t_{i}$ é igual ao valor latente não observado $t_{i}^{*}$ apenas quando um valor positivo de horas de trabalho é observado; caso contrário, tem-se o valor zero.
A função de máxima verossimilhança, para esse modelo, é:

$$
\begin{aligned}
& \mathrm{LL}=\sum_{0} \ln \left[1-F\left(X_{1 \mathrm{i}}^{\prime} \mathrm{b}_{1}\right)\right]+ \\
& +\sum_{+} \ln \left[F\left(\frac{X_{1 \mathrm{i}}^{\prime} \mathrm{b}_{1}+\frac{r}{\mathrm{~S}}\left(\mathrm{t}_{\mathrm{i}}-X_{2 \mathrm{i}}^{1} \mathrm{~b}_{2}\right)}{\sqrt{1-\mathrm{r}^{2}}}\right) \frac{1}{\mathrm{~S}} j\left(\frac{\mathrm{t}_{\mathrm{i}}-X_{2 \mathrm{i}}^{1} \mathrm{~b}_{2}}{\mathrm{~S}}\right)\right]
\end{aligned}
$$

Outro método de estimação conjunta é o modelo double hurdle, desenvolvido por Cragg (1971). Nesse modelo, o indivíduo precisa superar dois obstáculos (hurdles) para poder informar um número positivo de horas de trabalho não agrícola. $O$ primeiro obstáculo diz respeito à decisão de participação, e o segundo, ao nível dessa participação, isto é, à decisão de alocação de horas de trabalho.

O modelo double hurdle assemelha-se ao modelo de Heckman (1979) na medida em que reconhece dois estágios na decisão de alocação de horas de trabalho e também a utilização de diferentes variáveis explicativas nos dois processos de decisão. No entanto, diferente do de Heckman - que não admite a presença de informações com valores zero na segunda etapa -, o modelo double hurdle reconhece a possibilidade de haver zeros na segunda etapa, como resultado de escolhas deliberadas dos indivíduos ou de circunstâncias aleatórias. Isso significa que o indivíduo pode ser um potencial trabalhador, mas, por algum motivo, opta por não alocar horas de trabalho, como afirmam Cragg (1971) e Wodjao (2007). O modelo tem uma representação de uma variável latente, de forma que

$$
t_{i}= \begin{cases}t_{i}^{*} & \text { se } d_{i}=1 \text { e } t_{i}^{*}>0 \\ 0 & \text { caso contrário }\end{cases}
$$

O modelo double hurdle é apropriado quando as decisões de participação e de alocação de horas de trabalho são realizadas simultaneamente. $\mathrm{O}$ modelo mostra que o número de horas de trabalho alocadas pode ser zero, quando existe censura em zero $\left(t_{i}^{*} \leq 0\right)$ ou quando não há formações, ou devido à existência de alguma circunstância aleatória. De acordo com Gao et al. (1995) as condições em que se observa zero no modelo são dadas por: 
$\mathrm{t}_{\mathrm{i}}=\mathrm{t}_{\mathrm{i}}^{*}=\mathrm{X}_{2 \mathrm{i}}^{\prime} \mathrm{D}_{2}+\mathrm{v}_{\mathrm{i}}$ se

$\mathrm{X}_{1 \mathrm{i}}^{\prime} \mathrm{b}_{1}+\mathrm{m}_{\mathrm{i}}>0$ e $\quad \mathrm{X}_{2 \mathrm{i}}^{\prime} \mathrm{b}_{2}+\mathrm{v}_{\mathrm{i}}>0$

$t_{i}=t_{i}^{*}=X_{2 i}^{\prime} b_{2}+v_{i}$ se

$X_{1 \mathrm{i}}^{\prime} \mathrm{b}_{1}+\mathrm{m}_{\mathrm{i}}>0$ e $\quad \mathrm{X}_{2 \mathrm{i}}^{\prime} \mathrm{b}_{2}+\mathrm{v}_{\mathrm{i}} \leq 0$

$t_{i}=t_{i}^{*}=X_{2 i}^{\prime} b_{2}+v_{i}$ se

$X_{1 \mathrm{i}}^{\prime} \mathrm{b}_{1}+\mathrm{m}_{\mathrm{i}} \leq 0$ e $\quad \mathrm{X}_{2 \mathrm{i}}^{\prime} \mathrm{b}_{2}+\mathrm{v}_{\mathrm{i}}>0$

$\mathrm{t}_{\mathrm{i}}=\mathrm{t}_{\mathrm{i}}^{*}=\mathrm{X}_{\mathrm{2i}}^{\prime} \mathrm{b}_{2}+\mathrm{v}_{\mathrm{i}}$ se

$X_{1 i}^{\prime} b_{1}+m_{i} \leq 0$ e $\quad X_{2 i}^{\prime} b_{2}+v_{i} \leq 0$

Assim, números positivos da participação e de horas de trabalho são observados se um indivíduo participa do mercado de trabalho rural não agrícola e aloca determinada quantidade de horas de trabalho. Valores diferentes de zero também podem ser observados nas demais condições.

O modelo double hurdle, com erros independentes, pode ser estimado a partir da função de máxima verossimilhança:

$$
\begin{aligned}
& \mathrm{LL}=\sum_{0} \ln \left[1-\mathrm{F}\left(\mathrm{X}_{1 \mathrm{i}}^{\prime} \mathrm{b}_{1}\right) \mathrm{F}\left(\frac{\mathrm{X}_{2 \mathrm{i}}^{\prime} \mathrm{b}_{2}}{\mathrm{~S}}\right)\right]+ \\
& +\sum_{+} \ln \left[\mathrm{F}\left(\mathrm{X}_{1 \mathrm{i}}^{\prime} \mathrm{b}_{1}\right) \frac{1}{\mathrm{~S}} j\left(\frac{\mathrm{t}_{\mathrm{i}}-\mathrm{X}_{2 \mathrm{i}}^{\prime} \mathrm{b}_{2}}{\mathrm{~S}}\right)\right]
\end{aligned}
$$

A equação de participação no mercado de trabalho não agrícola dos pais e dos filhos pode ser representada pela seguinte equação:

$$
W_{i}=a_{0}+\sum_{j=1}^{j} a_{j} X_{j i}+d T_{i}+e
$$

em que $W_{i}$ representa a decisão de participação de pais e de filhos em atividades não agrícolas; $T_{i}$ é a variável dummy que indica se a família é beneficiária de programa de transferência de renda; $X_{\mathrm{ji}}$ representa as variáveis de controle - características individuais e da família.

A decisão de alocação de horas de trabalho pelos chefes de família, no modelo de Heckman (1979), pode ser representada pela seguinte equação:

$$
H_{i}=a_{0}+g T_{i}+\sum_{j=1}^{j} b_{j} X_{j i}+s l_{i}+e
$$

em que $\mathrm{H}_{\mathrm{i}}$ representa a quantidade de horas por semana alocadas no trabalho não agrícola; $\mathrm{T}_{\mathrm{i}}$ é a variável binária que indica se a família é beneficiária de programas de transferência de renda; os $X_{\mathrm{ji}}$ referem-se às características individuais e da família; e $\lambda_{i}$ é a inversa da "razão de Mills", também conhecida por "correção de seleção", derivada da decisão de participação, que pode ser obtida pela seguinte expressão:

$$
l_{i}=\frac{f(Z)}{1-F(Z)^{\prime}}
$$

em que $\square$ e $\square$ são, respectivamente, a função de densidade e a função de distribuição da variável normal padrão $Z_{\mathrm{i}}$.

No modelo duble-hurdle, a equação de horas de trabalho de pais e filhos é representada por

$$
\begin{aligned}
& H_{i}^{*}=a_{0}+g T_{i}+\sum_{j=1}^{j} b_{j} X_{j i}+e \\
& H_{i}= \begin{cases}H_{i}^{*} & \text { se } W_{i}=1 \\
0 \text { para qualquer outro de valor } w_{i}\end{cases}
\end{aligned}
$$

As informações referentes às decisões de participação e de alocação de horas de trabalho em atividades não agrícolas foram coletadas nos microdados da PNAD de 2006. Nesse ano, o Instituto Brasileiro de Geografia e Estatística (IBGE) divulgou, junto à pesquisa anual da PNAD, um suplemento com informações sobre o acesso das famílias aos programas de transferência de renda. Foram utilizados dois arquivos de dados: um com as informações de domicílios e outro com as informações sobre as pessoas residentes em área rurais. Na delimitação da área rural, empregou-se a definição do IBGE, que considera como área rural a área externa ao perímetro urbano de um distrito, compreendendo os seguintes tipos de localização: rural de extensão urbana, rural povoado, rural núcleo, aglomerado rural e rural exclusive aglomerado.

No sentido de agregar essas informações em unidades familiares, adotou-se o conceito de família extensiva, que inclui, além da família nuclear, os parentes que vivem no mesmo domicílio e os agregados. A partir dos dados coletados, usou-se 
o código da pessoa de referência na família - o chefe -, que residia em domicílio particular permanente, incluindo-se, no cômputo da família, o cônjuge, os filhos, os parentes e os agregados. Não entraram na composição da família os pensionistas, os empregados domésticos e nem os parentes de empregados domésticos.

O conceito de família empregado neste estudo é o mesmo que foi utilizado por Del Grossi e Graziano da Silva (1998) e, como tipologia de unidade familiar, em vários estudos do "Projeto Rurbano", uma ampla pesquisa realizada pelo Instituto de Economia da Unicamp, cujo objetivo era analisar as transformações no emprego rural brasileiro. Portanto, as informações referem-se às famílias extensivas pobres residentes em domicílios permanentes, em áreas rurais, que foram tratadas e as não tratadas pelos programas sociais e de transferência de renda. Para selecionar as famílias pobres utilizou-se a linha de pobreza do Bolsa Família vigente em 2006, que era de R\$120,00.
O Quadro 1 descreve as variáveis utilizadas na estimação das equações de participação e de alocação de horas de trabalho.

Na estimação da equação de participação e de alocação de horas de trabalho, foram utilizadas variáveis referentes às características individuais do chefe e de sua família. A partir do conjunto de dados, foram geradas as duas variáveis de resposta utilizadas nas equações de participação e de horas de trabalho. Na primeira equação, a variável dependente é uma variável binária, que capta a decisão de participação em atividades não agrícolas, e a segunda variável mensura o número de horas alocadas em atividades não agrícolas.

As variáveis explicativas referem-se às características dos pais e às dos filhos. Entre as características dos pais, a variável gênero foi utilizada no sentido de se verificar se o chefe de família homem tem mais chances de participação nas atividades não agrícolas do que a mulher que comanda a família. Espera-se que a chance de participação

Quadro 1. Descrição das variáveis utilizadas nas equações de participação e de alocação de horas de trabalho em atividades não agrícolas - PNAD, 2006

\begin{tabular}{|c|c|}
\hline Variáveis & Descrição \\
\hline \multicolumn{2}{|l|}{ Variáveis dependentes } \\
\hline Decisão de trabalhar & $\begin{array}{l}\text { em atividades não agrícolas }=1 ; \\
\text { em atividades agrícolas }=0\end{array}$ \\
\hline Horas trabalhadas & horas/semana em atividades não agrícolas \\
\hline \multicolumn{2}{|l|}{ Variáveis explicativas } \\
\hline \multicolumn{2}{|l|}{ Características individuais } \\
\hline Gênero & masculino $=1 ;$ feminino $=0$ \\
\hline Cônjuge & cônjuge $=1$; sem cônjuge $=0$ ) \\
\hline Raça & branca $=1$; não branca $=0$ \\
\hline Idade & Anos \\
\hline Experiência & anos ao quadrado \\
\hline Educação & anos de estudo \\
\hline Filho(s) até 05 & número de filhos até cinco anos \\
\hline Filho(s) 6 a10 & número de filhos de seis até dez anos \\
\hline \multicolumn{2}{|l|}{ Filho(s) 11 a 15} \\
\hline Empregado & empregado $=1$; Caso contrário $=0$ \\
\hline Adultos & número de adultos acima de 17 anos \\
\hline Condição na ocupação & conta própria $=1 ;$ caso contrário $=0$ \\
\hline Acesso à terra & com posse da terra $=1$; sem posse $=0$ \\
\hline \multicolumn{2}{|l|}{ Características da família } \\
\hline Bolsa Família - (BF + Programa de Erradicação do Trabalho Infantil) & recebe o $\mathrm{BF}$ e ou $\mathrm{PET}=1$; caso contrário $=0$ \\
\hline Benefício Prestação Continuada - BPC & recebe o $\mathrm{BPC}=1$; caso contrário $=0$ \\
\hline Aposentados & aposentados no domicílio $=1 ;$ caso contrário $=0$ \\
\hline
\end{tabular}

Fonte: Microdados da Pesquisa Nacional por Amostra de Domicílios, PNAD. Suplemento: acesso aos programas sociais, 2006. Elaboração dos autores. 
em atividades não agrícolas do chefe familiar, que tem um cônjuge, supere a chance de participação de famílias com apenas um membro parental.

Procurou-se analisar se a raça ou cor do chefe de família afeta a sua decisão de participação e de alocação de horas de trabalho em atividades não agrícolas. Com a inclusão dessa variável tentou-se captar possíveis diferenças na probabilidade de participação, em atividades não agrícolas, entre brancos e não brancos. Normalmente os estudos consideram o efeito da raça ou etnia, sobre a renda do trabalho, para captar o efeito discriminação ou segmentação. Por exemplo, Nery e Hoffmann (2009) observaram que o rendimento do trabalho principal das pessoas da cor preta ou parda e indígenas, em áreas rurais, era menor do que o rendimento do trabalho principal de pessoas de cor branca.

Por outro lado, Santos et al. (2010) na tentativa de verificar os fatores que determinam a participação de homens e de mulheres no mercado de trabalho, no meio rural brasileiro, analisaram, além da raça, o gênero, o nível de escolaridade da pessoa, consideram a localização geográfica do domicílio. Mesquita et al. (2010) também verificaram o efeito da raça sobre a probabilidade de participação das pessoas nas ocupações não agrícolas. Os autores constataram que os trabalhadores não brancos têm maior probabilidade de participação nas atividades não agrícolas no meio rural brasileiro e no nordestino.

No presente estudo, o efeito da raça sobre a decisão de participar e de alocar horas de trabalho não agrícola será captada por meio da variável dummy (Raça), controlando-se para as pessoas brancas.

Utilizou-se a variável idade no sentido de verificar se as pessoas com mais idade têm mais chances de participação no trabalho não agrícola e de alocação de horas nesse trabalho. Procurou-se captar, por meio da variável idade ao quadrado, o efeito da experiência do indivíduo sobre sua chance de participação e de alocação de horas. Esperava-se que as pessoas mais experientes tivessem mais chances de participação e de alocação de horas de trabalho.
A variável educação, mensurada pelo número de anos de estudos, foi utilizada no sentido de verificar se as pessoas com níveis mais elevados de educação têm mais chances de participar de atividades não agrícolas e de alocar horas de trabalho.

Com a condição do empreendimento agrícola, foi possível verificar o acesso à terra por parte do chefe da família. Espera-se que o acesso à terra, seja como posseiro, arrendatário ou proprietário, influencie na decisão do chefe da família de participação e de alocação de horas de trabalho em atividades não agrícolas. Para Schneider (2009), os indivíduos que formam a família podem optar entre combinar duas ou mais ocupações - assumindo a condição de famílias pluriativas - ou escolher pela troca de ocupação, deixando o trabalho agrícola e passando a ocupar-se em atividades não agrícolas.

Procurou-se, também, verificar a relação existente entre os trabalhadores ocupados e a oferta de trabalho não agrícola. Essa relação também foi objeto de pesquisa em muitos trabalhos do Projeto Rurbano. Por exemplo, Del Grossi et al. (2001) constataram que a renda média dos trabalhadores por conta própria, ocupados em atividade não agrícola, é maior do que a dos ocupados em atividades agrícolas.

A condição da ocupação do chefe da família em trabalho principal não agrícola permite classificá-lo como por conta própria, empregado ou empregador. Foram utilizadas as duas primeiras variáveis no sentido de averiguar os efeitos na decisão de alocação de horas de trabalho nas atividades não agrícolas.

Em relação aos programas de transferência, Bolsa Família e Benefício de Prestação Continuada, procurou-se examinar possíveis efeitos de desestímulo desses programas na estrutura de participação e de alocação de horas de trabalho dos pais e dos filhos em atividades não agrícolas. Foram levadas em consideração, na captação do efeito Bolsa Família, as respostas do questionário da PNAD de 2006 referentes às seguintes perguntas: se havia algum morador recebendo dinheiro do programa Bolsa Família; e, 
se algum morador recebia dinheiro do Programa de Erradicação Infantil (Peti). Considerando-se, que esses programas foram unificados e, em atenção ao terceiro eixo do programa Bolsa Família, que trata da vulnerabilidade e da inserção social, optou-se por reunir as respostas em uma única variável dummy, Bolsa Família.

O recebimento de aposentadorias por um dos membros da família também foi utilizado para captar o efeito renda negativo sobre a decisão de participação e de alocação de horas de trabalho não agrícola. A intensão era verificar se, por meio do efeito indireto, ou seja, com o recebimento de benefício dos aposentados, ocorre desestímulo ao trabalho de jovens e de chefes de família. Quanto à participação das aposentadorias na renda das famílias, Graziano da Silva (2001) considera que uma parcela da produção de subsistência é financiada pelas transferências sociais, sendo a principal delas a proveniente da aposentadoria, que responde a uma parte significativa das rendas monetárias das famílias rurais no Nordeste.

Os estudos de Delgado e Cardoso Júnior (2001), Maia Gomes (2001), Hoffmann (2003) e Ferreira e Souza (2007) mostram a importância dos recursos da Previdência Social como alternativa de renda para as famílias mais pobres, na região rural do Nordeste, e a importância desses benefícios sobre os indicadores de pobreza e desigualdade de renda. Por exemplo, Mariano e Lopes (2009) constataram que cerca de 30\% dos rendimentos das famílias rurais da região Nordeste são provenientes dos benefícios de aposentadorias e pensões.

Se esses benefícios garantem uma renda mínima para as famílias pobres, a questão passa a ser, portanto, verificar se esse efeito impactaria negativamente na decisão dos demais membros da família - pais e filhos - sobre a participação e alocação de horas de trabalhos em atividades não agrícolas.

Para examinar o efeito da presença de filhos sobre a oferta de trabalho não agrícola dos pais foram utilizadas três variáveis, que captaram o número de filhos por faixa etária - filhos de até 5 anos, de 6 a 10 anos e de 11 a 15 anos. Até 2006, o Bolsa Família tinha como focalização as família pobres com crianças e jovens até 15 anos de idade. Em 2008, ocorreram modificações no desenho do programa, com a inclusão de jovens de 16 e 17 anos. Assim, neste estudo, considerou-se, na análise, apenas o grupo etário de crianças e jovens até 15 anos. Esperavam-se impactos distintos da presença de filhos, por faixa etária, na oferta de trabalho dos pais. Por exemplo: na presença de filhos mais novos, de até 5 anos, os pais podem decidir reduzir as horas de trabalho em outras atividades, pois terão de alocar o tempo disponível com o trabalho e os cuidados com os filhos menores. Entretanto, quando existem filhos com idade mais elevada, os pais têm mais tempo para ofertar horas de trabalho.

\section{Resultados}

Nesta seção, são apresentados os resultados das estimações das equações de participação em atividades não agrícolas e de alocação de horas nessas atividades, com base nos modelos de Heckman e double hurdle. Inicialmente, estimou-se a influência dos programas sociais e de transferência de renda sobre a decisão de participação e a de alocação de horas, utilizando-se a amostra dos chefes de família e, posteriormente, a amostra dos filhos com idade de 11 a 15 anos. A Tabela 3 contém os resultados das estimações dos modelos de Heckman (1975) e duble-hurdle, de Cragg (1971), para as equações de participação e de alocação de horas de trabalho em atividades não agrícolas de chefes de família.

O coeficiente do parâmetro da razão de Mils (l) foi significativo a 5\%, mostrando, assim, a importância do procedimento de Heckman para corrigir o viés de seleção. Na estimação da equação de participação, o coeficiente da variável gênero apresentou sinal positivo, indicando que os chefes homens estão mais propensos a participar das atividades não agrícolas do que as mulheres chefes.

A variável raça não se revelou estatisticamente significativa, não sendo possível, portanto, 
082 - Efeitos dos Programas de Transferência de Renda sobre a Oferta de Trabalho Não Agrícola na Área Rural da Região Nordeste

Tabela 3. Estimações das equações de participação e de alocação de horas de trabalho dos chefes de família em atividades não agrícolas - Nordeste rural, 2006

\begin{tabular}{|c|c|c|c|c|}
\hline \multirow[b]{2}{*}{ Variáveis } & \multicolumn{2}{|c|}{ Modelo de Heckman } & \multicolumn{2}{|c|}{ Modelo de Cragg - double hurdle } \\
\hline & $\begin{array}{c}\text { Decisão de } \\
\text { participação }\end{array}$ & $\begin{array}{c}\text { Alocação de } \\
\text { horas }\end{array}$ & $\begin{array}{c}\text { Decisão de } \\
\text { participaçãao }\end{array}$ & $\begin{array}{c}\text { Alocação de } \\
\text { horas }\end{array}$ \\
\hline \multirow{2}{*}{ Cônjuge } & $-0,0779$ & 2,564 & $-0,0805$ & 2,6994 \\
\hline & $(0,113)$ & $(2,450)$ & $(0,113)$ & $(2,566)$ \\
\hline \multirow{2}{*}{ Gênero } & $0,3322^{* * *}$ & $12,150^{* * *}$ & $0,3125^{* * *}$ & $12,538^{* * *}$ \\
\hline & $(0,114)$ & $(2,779)$ & $(0,115)$ & $(2,857)$ \\
\hline \multirow{2}{*}{ Raça branca } & 0,0397 & $-1,58$ & 0,0413 & $-1,6654$ \\
\hline & $(0,069)$ & $(1,414)$ & $(0,0697)$ & $(1,464)$ \\
\hline \multirow{2}{*}{ Idade } & $0,0635^{* * *}$ & $0,5996^{*}$ & $0,062^{* * *}$ & $0,6442^{* *}$ \\
\hline & $(0,016)$ & $(0,352)$ & $(0,016)$ & $(0,345)$ \\
\hline \multirow{2}{*}{ Idade $^{2}$} & $-0,0008^{* * *}$ & $-0,0097^{* *}$ & $-0,0007^{* * *}$ & $-0,0103^{* * *}$ \\
\hline & $(0,0001)$ & $(0,004)$ & $(0,0002)$ & $(0,0041)$ \\
\hline \multirow{2}{*}{ Educação } & $0,0845^{* * *}$ & $-0,3181$ & $0,0852^{* * *}$ & $-0,3487^{*}$ \\
\hline & $(0,0001)$ & $(0,2416)$ & $(0,01)$ & $(0,195)$ \\
\hline \multirow{2}{*}{ Filhos até 5 anos } & 0,0189 & $1,2680^{*}$ & 0,0184 & 1,2551 \\
\hline & $(0,034)$ & $(0,726)$ & $(0,034)$ & $(0,751)$ \\
\hline \multirow{2}{*}{ Filhos 6 a 10 anos } & 0,031 & $-0,9326$ & 0,0322 & $-0,9683$ \\
\hline & $(0,035)$ & $(0,736)$ & $(0,035)$ & $(0,757)$ \\
\hline \multirow{2}{*}{ Filhos 11 a 15 anos } & $0,0911^{* *}$ & $1,6888^{* *}$ & $0,0894^{* *}$ & $1,7993^{* * *}$ \\
\hline & $(0,038)$ & $(0,835)$ & $(0,038)$ & $(0,853)$ \\
\hline \multirow{2}{*}{ Número de adultos } & $0,0721^{* *}$ & 0,0137 & $0,0717^{* *}$ & 0,0397 \\
\hline & $(0,032)$ & $(0,721)$ & $(0,032)$ & $(0,748)$ \\
\hline \multirow{2}{*}{ Bolsa Família com o Peti } & $-0,1663^{* * *}$ & $-0,7117$ & $-0,1653^{* * *}$ & $-0,7945$ \\
\hline & $(0,060)$ & $(1,326)$ & $(0,060)$ & $(1,300)$ \\
\hline \multirow{2}{*}{$\mathrm{BPC}$} & $-0,8645^{* *}$ & $-3,7899$ & $-0,8659^{* *}$ & $-3,8586$ \\
\hline & $(0,345)$ & $(10,00)$ & $(0,347)$ & $(10,37)$ \\
\hline \multirow{2}{*}{ Aposentados } & $-0,3827^{* *}$ & $-9,0606^{* *}$ & $-0,3531^{* *}$ & $-10,3667^{* * *}$ \\
\hline & $(0,177)$ & $(4,395)$ & $(0,182)$ & $(4,823)$ \\
\hline \multirow{2}{*}{ Posse da terra } & $-1,6246^{* * *}$ & - & $-1,6255^{* * *}$ & 0,4767 \\
\hline & $(0,102)$ & & $(0,1028)$ & $(3,588)$ \\
\hline \multirow{2}{*}{ Conta própria } & - & $-3,0161$ & & $-2,1365$ \\
\hline & & $(1,939)$ & & $(2,077)$ \\
\hline \multirow{2}{*}{ Empregado } & & 0,733 & & 1,5319 \\
\hline & & $(1.971)$ & & $(2,112)$ \\
\hline
\end{tabular}

Wald chi2(14) $=722.16$

Prob $>$ chi $2=0.0000 \quad$ log likelihood: -1369.66

Heckman lambda

$\lambda=-0.212(0.027)$

Erros padrão entre parênteses; $* * *, * * \mathrm{e}^{*}$ : significativo a $1 \%, 5 \%$ e $10 \%$, respectivamente.

Fonte: Dados da PNAD 2006. Elaboração dos autores.

verificar efeitos de discriminação na decisão de participação e de alocação de horas. Resultado semelhante foi encontrado por Santos et al. (2010), que concluíram, que não haveria discriminação no mercado de trabalho rural brasileiro.

Notou-se também que a decisão dos chefes de família de participar de atividades não agríco- las depende da idade deles, ou seja, os chefes de família com idade mais elevada têm mais chances de estarem envolvidos em atividades não agrícolas.

A experiência, mensurada pela proxi (idade) ${ }^{2}$, apresentou sinal negativo, indicando queda na produtividade do trabalho e, assim, da partici- 
pação de pessoas mais idosas em atividades não agrícolas.

A educação mostrou-se um atributo importante na decisão de participação do chefe de família de trabalhar em atividades não agrícolas. $\mathrm{O}$ sinal positivo e significativo na equação de participação permite observar que as chances dos chefes de família aumentam com os anos de estudo.

Os resultados da estimação da equação de participação revelaram que as chances dos chefes de família de participar de atividades não agrícolas são maiores quando, na composição da família, existem filhos na faixa etária entre 11 e 15 anos.

A existência de membros adultos no domicílio também afeta positivamente a decisão dos chefes de família de participar de atividades não agrícolas. A presença de outros adultos na família pode gerar mais confiança para os pais ausentarem-se de seus domicílios ao se engajarem em atividades não agrícolas que, em muitos casos, são desenvolvidas fora da unidade domiciliar.

Os coeficientes das variáveis que identificam o acesso das famílias aos programas sociais e de transferência condicional mostraram-se significativos e com sinal negativo, indicando, assim, que os chefes de famílias pobres que são atendidos pelos programas Bolsa Família e Benefício de Prestação Continuada e pelas aposentadorias têm menos chances de se ocupar em atividades não agrícolas. Nesse sentido, o acesso a esses programas desestimularia a participação dos chefes de famílias em ocupações não agrícolas.

$\mathrm{O}$ acesso à terra revelou-se também uma variável importante na decisão de participação dos chefes de famílias nas ocupações não agrícolas: ou seja, a posse da terra, seja na forma de proprietário, arrendatário ou posseiro, diminui as chances dos chefes de família de participar de outras atividades fora da agricultura. A posse da terra permite que o agricultor tenha maior dedicação às ocupações agrícolas, entre elas o cuidado no plantio, no cultivo, na colheita e na comercialização das culturas.

Esse resultado está em conformidade com o pensamento de Ney e Hoffmann (2008), que per- ceberam que o nível de renda não agrícola das famílias rurais deve estar negativamente correlacionado com a posse da terra; ou seja, que as famílias rurais, que sofrem com a escassez de terras, têm maior dependência do rendimento das atividades não agrícolas se comparadas às famílias com maior disponibilidade de terras. Entretanto, quanto à relação entre nível de renda em atividades não agrícolas e posse da terra a relação encontrada por esses autores foi positiva.

Essa associação foi também discutida por Buainain et al. (2003), os quais observaram que as atividades não agrícolas se apresentam como uma das oportunidades de trabalho, em geral precária, para os membros de famílias com pouco acesso ao progresso técnico, ao crédito, à assistência técnica e à terra.

É importante lembrar, que esse resultado não considera o tamanho da propriedade. De acordo com Schneider (2009), as famílias pluriativas possuem terras com menores áreas e cultivam uma superfície agrícola média menor do que as famílias exclusivamente agrícolas. Ou seja, a posse da terra pode não refletir totalmente a decisão de trabalhar em atividades não agrícolas.

Em relação à estimação da equação de alocação de horas de trabalho do modelo de Heckman, os resultados da estimação revelam que o número de horas alocadas nas atividades não agrícolas é maior para os chefes de família homens do que para os chefes mulheres. Tal como na decisão de participação, os chefes de família tendem a alocar mais horas de trabalho nas ocupações não agrícolas à medida que ficam mais adultos, ou seja, chefes de família com idade mais elevada tendem a ofertar mais horas de trabalho do que os mais jovens. A variável idade ao quadrado, que capta a não linearidade da idade, também apresentou a mesma relação quanto à decisão de participação, a saber, que as pessoas mais idosas, em decorrência da queda na produtividade, tendem a diminuir o número de horas de trabalho alocadas em atividades não agrícolas.

Os chefes de família também tendem a aumentar o número de horas alocadas em ocupações não agrícolas, quando na composição da 
família há filhos menores de 5 anos e na faixa etária de 11 a 15 anos. A presença de filhos menores pode afetar a decisão dos pais de oferecerem mais horas de trabalho fora das atividades agrícolas quando novas fontes de renda forem necessárias para suprir a renda familiar. No entanto, a presença de filhos com mais de 11 anos pode gerar confiança para os pais deixarem os filhos menores aos cuidados dos maiores, quando há a necessidade de trabalhar fora da propriedade familiar.

De acordo com os resultados da equação de alocação de horas de trabalho no modelo de Heckman a presença de aposentados na família influencia, negativamente, a decisão de alocação de horas de trabalho em atividades não agrícolas; isto é, com a renda dos membros aposentados, a família garantiria uma renda mínima de sobrevivência sem o trabalho fora da atividade agrícola.

Quanto à decisão de participação em atividades não agrícolas, os resultados do modelo double hurdle sugerem que os chefes de família homens têm mais chances de participação do que as mulheres. Essa maior participação dos chefes homens pode estar associada a ocupações rurais não agrícolas, que demandam mão de obra masculina, como as dos setores da indústria e da construção civil. Na equação de alocação de horas de trabalho, o efeito da presença do chefe masculino também se repete. Ou seja, os chefes de família do gênero masculino ofertam mais horas de trabalho em atividades não agrícolas. $\mathrm{O}$ efeito da idade ao longo do tempo foi semelhante aos resultados encontrados no modelo de Heckman. Ou seja, com o aumento da idade, os chefes de família têm mais chances de participar de ocupações não agrícolas e de alocar mais horas de trabalho; mas, para os chefes mais idosos, o efeito da idade atua em sentido contrário na chance de participação e na de alocação de horas de trabalho.

A decisão dos pais de participar de ocupações não agrícolas é influenciada pelo seu nível de escolaridade; ou seja, quanto mais anos de escola, maiores as chances de participação nas ocupações não agrícolas. Entretanto, o capital humano obtido com os anos de estudo apresentou efeito contrário na equação de alocação; isto é, os chefes com maior grau de instrução tendem a diminuir a quantidade de horas de trabalho alocadas em atividades não agrícolas.

A presença de filhos no domicílio também se mostrou capaz de influenciar a participação e a decisão de alocação de trabalho rural não agrícola dos chefes de família. Isto é, os pais com criança(s) na faixa de etária entre 11 e 15 anos são mais propensos a participar de atividades não agrícolas, bem como a ofertar mais horas de trabalho nessas atividades. Os resultados da estimação do modelo double hurdle revelam que as chances de os pais participarem de atividades não agrícolas também são maiores quando há adultos na composição da família.

Os resultados das estimações das equações de participação e alocação mostraram que o acesso aos programas Bolsa Família e Benefício de Prestação Continuada e às aposentadorias causa efeito negativo sobre as chances de participação dos chefes de família em ocupações não agrícolas. $\mathrm{O}$ acesso de algum membro da família ao benefício das aposentadorias também contribui para que os chefes de família reduzam o número de horas de trabalho alocadas em atividades não agrícolas. Notou-se, também, que a posse de terra contribui para a redução das chances de os chefes de família participarem de atividades não agrícolas.

Os resultados encontrados com o modelo double hurdle são semelhantes àqueles observados no estudo de Mesquita et al. (2009), que empregaram esse modelo para analisar a decisão de participação e alocação de horas de trabalho não agrícola. Entre esses resultados estão maior participação e alocação de trabalho dos homens chefes de família com maior grau de escolaridade.

Na Tabela 4, são apresentados os resultados das estimações do modelo double hurdle das equações de participação e de alocação de horas de trabalho de filhos na faixa etária de 10 a 15 anos. A análise desse grupo de tratamento é relevante por ele ter influência significativa na oferta de trabalho dos demais membros do domicílio. Ainda foram considerados nas estimações os efeitos de variáveis de controle associadas aos pais nas 
Tabela 4. Estimações do modelo double hurdle do efeito dos programas de transferência de renda sobre a oferta de trabalho não agrícola de filhos de 10 a 15 anos de idade - Nordeste rural, 2006

\begin{tabular}{|c|c|c|}
\hline \multirow{2}{*}{ Variáveis } & \multicolumn{2}{|c|}{ Modelo double hurdle } \\
\hline & Equação de participação & Equação de horas de trabalho \\
\hline \multirow{2}{*}{ Bolsa Família } & $-1,3983^{* *}$ & 3,1778 \\
\hline & $(0,716)$ & $(7,57)$ \\
\hline \multirow{2}{*}{ BPC /Aposentados } & 1,9103 & $-21,855$ \\
\hline & $(1,848)$ & $(15,10)$ \\
\hline \multirow{2}{*}{ Gênero filhos } & 0,0092 & $-16,237^{* * *}$ \\
\hline & $(0,589)$ & $(6,80)$ \\
\hline \multirow{2}{*}{ Idade filhos } & $0,4848^{* * *}$ & $-1,639$ \\
\hline & $(0,174)$ & $(2,520)$ \\
\hline \multirow{2}{*}{ Anos de estudo } & $-0,3901^{* *}$ & $6,8191^{* * *}$ \\
\hline & $(0,199)$ & $(1,972)$ \\
\hline \multirow{2}{*}{ Idade do pai } & $-0,0089$ & $0,4476^{* *}$ \\
\hline & $(0,016)$ & $(0,242)$ \\
\hline \multirow{2}{*}{ Anos de estudo pai } & 0,112 & $-0,2005$ \\
\hline & $(0,137)$ & $(1,234)$ \\
\hline \multirow{2}{*}{ Diferença idades entre pai e mãe } & $-0,5511^{* * *}$ & $0,8897^{* * *}$ \\
\hline & $(0,022)$ & $(0,277)$ \\
\hline \multirow{2}{*}{ Anos de estudo da mãe } & $-0,1827^{*}$ & $2,8087^{* * *}$ \\
\hline & $(0,105)$ & $(1,296)$ \\
\hline \multirow{2}{*}{ Número de filhos } & $-1,0206^{* * *}$ & 8,1867 \\
\hline & $(0,275)$ & $(5,349)$ \\
\hline Acesso à terra & $\begin{array}{c}-1,2193^{* * *} \\
(0,569)\end{array}$ & \\
\hline Empregado & & $\begin{array}{c}55,568^{* * *} \\
(10,35)\end{array}$ \\
\hline Log likelihood $=-750.5055$ & & \\
\hline
\end{tabular}

Erros padrão entre parênteses ***,** $\mathrm{e}^{*}$ : significativo a 1\%,5\% e 10\%, respectivamente.

Fonte: Dados da PNAD 2006. Elaboração dos autores.

equações de participação e alocação de horas de trabalho.

Na primeira equação, os resultados mostraram que, se a família está enquadrada no Bolsa Família e recebendo esse benefício, as chances de os jovens estarem ocupados em atividades não agrícolas diminuem. Esse resultado leva a implicações bastante significativas, pois o recebimento do benefício por esse grupo pode representar eficácia das metas do programa; ou seja, ele estaria erradicando o trabalho infantil e aumentando a frequência escolar de jovens. Na equação de alocação, o coeficiente do programa não foi significativo. Esses resultados são semelhantes aos encontrados por Cardoso e Souza (2004), os quais perceberam que os benefícios do Bolsa Família aumentam as chances de as crianças estu- darem, embora não reduzam as chances de estarem trabalhando.

O coeficiente negativo associado à variável gênero indica que, uma vez que participam de atividades não agrícolas, os jovens entre 10 e 15 anos do gênero masculino tendem a alocar menos horas de trabalho que as jovens.

O coeficiente da variável idade desse grupo de tratamento apresentou efeito positivo, indicando que os jovens com mais idade têm mais chances de participar de atividades não agrícolas. Já o coeficiente da variável educação dos filhos apresentou sinal negativo sobre a decisão de participação em atividades não agrícolas. Uma das explicações para esse resultado seria uma relação com a frequência escolar. Os jovens com mais anos de estudo tendem a frequentar mais 
a escola e a reduzir sua participação em atividades não agrícolas. Entretanto, se os jovens já estão ocupados em atividades não agrícolas, a tendência é que ofereçam mais horas de trabalho nessas atividades à medida que aumente seu nível de educação.

Os jovens também tendem a oferecer mais horas de trabalho não agrícola quando são filhos de pais mais idosos, mas a diferença de idade entre os pais também pode influenciar na decisão de participação e de alocação de horas de trabalho dos jovens. Na equação de participação, essa variável apresentou efeito negativo, indicando que, quanto maior a diferença de idade entre pai e mãe, menores são as chances de participação dos jovens nas atividades não agrícolas. Por outro lado, na equação de alocação, essa variável apresentou efeito positivo sobre a decisão dos jovens de alocar mais horas nessas atividades. Isto é, grande diferença de idade entre marido e mulher poderia induzir maior iniciativa dos filhos a buscarem ocupação para complementar a renda familiar.

A estimação da equação de participação mostrou efeito negativo dos anos de estudo da mãe sobre a decisão de participação dos filhos. Esse resultado sugere que as mães com maior escolaridade tendem a manter os filhos por mais tempo na escola, aumentando a frequência escolar destes e diminuindo sua participação em atividades não agrícolas. Entretanto, estando os jovens já ocupados em atividades não agrícolas, o grau de escolaridade das mães contribuiria para o aumento de horas trabalhadas dos filhos nas ocupações não agrícolas.

A decisão sobre a participação dos jovens em ocupações não agrícolas também pode ser influenciada pelo número de irmãos. O resultado da equação de participação apresentou sinal negativo, contrário ao esperado, pois, das famílias com grande número de filhos, poder-se-ia esperar uma tendência dos jovens a buscarem ocupações fora da agricultura. Esse resultado poderia ser explicado pela decisão do jovem de colaborar nas diversas atividades agrícolas junto aos demais membros da família.
No que tange à posição na ocupação do trabalho principal, a variável empregado apresentou efeito positivo, mostrando que os jovens ocupados em atividades não agrícolas, na condição de empregados, alocam mais horas de trabalho. $\mathrm{O}$ acesso à terra também pode afetar a decisão de participação dos filhos em atividade não agrícola. O sinal negativo dessa variável indica que os jovens com idade entre 10 e 15 anos têm as chances de participação em atividades não agrícolas reduzidas quando seus pais têm acesso à terra. Possivelmente, esse resultado está associado à tendência de participação dos filhos nas atividades da propriedade rural familiar, ao invés de procurarem ocupações fora do empreendimento agrícola.

\section{Considerações finais}

Este estudo procurou analisar o efeito das transferências de renda na decisão de oferta de trabalho rural não agrícola na região Nordeste, usando como estratégia a estimação de procedimentos econométricos que pudessem captar as decisões de participação e de oferta de horas de trabalho de pais e filhos em condição de pobreza.

No meio rural, um elemento que tem se destacado como componente importante da renda das famílias são as transferências de renda dos programas sociais, sobretudo no contexto dos programas Bolsa Família e BPC e dos benefícios das aposentadorias e pensões. Esses rendimentos têm se constituído em parte importante das rendas rurais, principalmente das famílias mais pobres e, sobretudo, no meio rural do Nordeste.

Os resultados, em geral, apontaram efeito negativo dos programas de transferência de renda e dos programas sociais sobre a decisão dos pais e dos filhos de participarem de atividades não agrícolas. No que diz respeito aos pais, esses efeitos devem ser interpretados separadamente: o recebimento do Bolsa Família exige maior atendimento às condições requeridas pelo programa, como a frequência escolar dos filhos, a atenção à saúde das crianças, entre outras, que demandam 
tempo e maior responsabilidade dos pais. Porém, surgem as seguintes questões: o valor recebido é suficiente para os pais tomarem a decisão de não participar de atividades não agrícolas? Ou seja, o efeito renda do programa é suficiente para essa tomada de decisão?

As respostas a essas questões não podem ser dadas sem se levar em consideração outras variáveis que afetam a decisão de participação e de alocação de horas de trabalho de uma pessoa em condições de pobreza que está vivendo no meio rural do Nordeste.

É importante lembrar que outros estudos, como o de Teixeira (2009) e o de Fogel e Barros (2010), também encontraram relação negativa entre a decisão de participação na oferta de trabalho e o acesso ao Bolsa Família. No entanto, perceberam que o Bolsa Família reduz marginalmente a oferta de horas de trabalho e que sua intensidade depende do valor da transferência.

Além disso, é importante investigar o acesso a outras fontes de renda - por exemplo: aos rendimentos das aposentadorias e do Benefício de Prestação Continuada. Esses benefícios chegam a representar mais de $30 \%$ da renda das famílias do meio rural do Nordeste e, para muitas famílias pobres, é sinônimo de sobrevivência de seus componentes, principalmente durante os períodos de seca e de entressafra.

Em relação às demais variáveis, os resultados mostraram que a educação influencia a decisão dos pais e a dos filhos de participarem de atividades não agrícolas; ou seja, as chances de participação crescem com os anos de estudo. A diversidade das atividades não agrícolas no meio rural possibilita oportunidades de ocupação que requerem níveis maiores de instrução. Observou-se, ainda, que os chefes de família pobres que de alguma forma dispõem da posse da terra, têm reduzidas suas chances de participação em atividades não agrícolas. $\mathrm{O}$ acesso à terra estimularia o chefe de família a dedicar mais tempo de trabalho às atividades do campo, necessárias para o melhor uso da propriedade, e para obter, do uso da terra, os recursos necessários à sobrevivência da família. Ou seja, para os chefes de família que dispõem da posse da terra, as chances de participação em atividades não agrícola se tornam reduzidas, ou sua dedicação às atividades agrícolas se torna maior. Lembrando que essa relação depende também do tipo da posse da terra e da área da propriedade.

Por outro lado, os resultados do estudo apontam na direção da eficácia do programa Bolsa Família em retirar os jovens do trabalho infantil, penoso e, em geral, não assistido pelos direitos trabalhistas. O efeito do Bolsa Família de reduzir as chances de participação dos jovens em atividades não agrícolas também pode estar associada à exigência da frequência escolar. De fato, os resultados do estudo mostraram que, para essa faixa etária, mais anos de estudo não aumentam as chances de participação nessas atividades, embora, no caso daqueles que já estão no mercado de trabalho, os anos de estudo contribuem para o aumento de horas de trabalho nessas ocupações.

Esses resultados permitem algumas reflexões sobre a eficácia dos programas sociais e de transferência de renda. Ao afetarem as decisões sobre a oferta de trabalho em atividades não agrícolas, esses programas podem criar condições para que os jovens atualmente em condições de pobreza no meio rural do Nordeste consigam quebrar o ciclo vicioso da pobreza geracional de suas famílias, por meio de melhor educação e formação, o que lhes possibilitará o acesso ao mercado de trabalho não agrícola em melhores condições que as que atualmente seus pais encaram.

\section{Referências bibliográficas}

ARISTEI, D. e PIERONI, L. A double-hurdle approach to modelling tobacco consumption in Italy. Applied Economics, v. 1, n. 1, abr. 2011.

BUAINAIN, A. M., ROMEIRO, A. R. e GURNZIROLI, C. Agricultura familiar e o novo mundo rural. Sociologias, Porto Alegre, ano 5, n. 10, jul./dez. 2003.

CARDOSO, E. e SOUZA, A. O. The impact of cash transfer on child labor and scholl attendance in Brazil. Working Paper, n. 4-w07, abr. 2004. Department of Economics Vanderbilt University. Disponivel em: <https://ideas.repec.org/p/van/wpaper/0407.html>. Acesso em: 15 out. 2012. 
CRAGG, J. G. Some Statistical Models for Limited Dependent Variables with Application to the Demand for Durable Goods, Econometrica, v. 39, 1971.

DAVIS, J. R. The Rural Non-Farm Economy, livelihoods, and their diversification: issues and options. Chatham, UK: Natural Resources Institute publication, 2004.

DELGADO, G. e CARDOSO JUNIOR, J. C. A universalização dos direitos sociais no Brasil: a previdência rural nos anos 90: transformações da agricultura e políticas públicas. Brasília, DF: IPEA, p. 418-444, 2001.

DEL GROSSI, M. E. e GRAZIANO SILVA, J. O novo rural brasileiro. Nova Economia, UFMG, v. 7, n. 1, p. 43-81, 1998.

. e GRAZIANO SILVA, J. Ocupações e rendas rurais no Brasil. In: Ocupações rurais não agrícolas. Oficina de Atualização Temática 2000, Londrina. Anais... Londrina: IAPAR, 2000.

., GRAZIANO DA SILVA, J., PEREIRA, C. F. e CAMPONHOLA, C. Diferencial de renda entre ocupações no meio rural brasileiro. In: CONGRESSO DA SOCIEDADE BRASILEIRA ECONOMIA, ADMINISTRAÇÃO E SOCIOLOGIA RURAL, 39, 2001, Recife (PE). Anais... Recife, 2001, p. 142.

. e GRAZIANO SILVA, J. Mudanças recentes no mercado de trabalho rural. Brasília, UnB, 2006.

DETRE, J. D., MARJ, J. T., MISHRA, A. K. e ADHIKARI, A. Linkage between direct marketing and farm income: a double approach. Agribusiness, v. 27, n. 1, p. 19-23, 2011.

FERREIRA, C. R. e SOUZA, S. C. I. As aposentadorias e pensões e a concentração dos rendimentos domiciliares per capita no Brasil e na sua área rural:1981 a 2003. Revista de Economia e Sociologia Rural, v. 45, n. 4, p. 9851.011, dez. 2007.

FERREIRA, F. e LANJOUW, P. Rural nonfarm activities and poverty in the Brazilian Northeast, World Development, v. 29, n. 3, edición especial, Amsterdam, Elsevier Science, 2001.

FERRO, A. R. e NICOLLELA, A. C. The impact of conditional cash transfers programs on household working decision in Brazil. São Paulo, 2007.

FERRO, A. R. e KASSOUF, A. L. Avaliação do impacto do Programa Bolsa-Escola sobre o trabalho infantil no Brasil. Pesquisa e Planejamento Econômico, v. 35, n. 3, p. 417-444, 2005.

FOGEL, M. N. e BARROS, R. P. Effects of conditional cash transfer programmers on adult labor supply: an empirical analysis using a time series cross section sample of brazilian municipalities. Revista Estado e Economia, v. 40, n. 2, p. 259-293, abr.jun. 2010.

GAO, X. M., WAILES, E. J. e CRAMER, G. L. Doublehurdle model with bivariate normal errors: an application to U.S. rice demand. Journal of Agricultural and Applied Economics, 1995.

GOMES DA SILVA, A. Novas configurações no espaço rural do Rio Grande do Norte. In: CAMPANHOLA, C. e GRAZIANO DA SILVA, J. (Orgs.). O novo rural brasileiro: uma análise estadual - Nordeste. Jaguariúna, SP: EMBRAPA, 2000.

GRAZIANO SILVA J. Velhos e novos mitos do rural brasileiro. Estudos Avançados, v. 15, n. 43, p. 37-50, 2001.

HECKMAN, J. Sample Selection Bias as a Specification Error. Econometrica, v. 47, n. 1, p. 153-161, 1979.

HOFFMANN, R. Inequality in Brazil: the contribution of pensions. Revista Brasileira de Economia, Rio de Janeiro, v. 57, n. 4, p. 755-773, out./dez. 2003.

Transferência de renda e a redução da desigualdade no Brasil e cinco regiões entre 1997 e 2004. Econômica, v. 8, n. 1, p. 55-81, 2006.

KLEIN, E. El empleo rural no agrícola en América Latina. Santiago: PREALC/OIT, 1992.

NEDER, H. D. e SILVA, J. L. M. Pobreza e Distribuição de Renda em Áreas Rurais: uma Abordagem de Inferência. Revista de Economia e Sociologia Rural, Rio de Janeiro, v. 42, n. 03, p. 469-486, jul./set. 2004.

NEY, M, G. e HOFMANN, R. A contribuição das atividades agrícolas e não agrícolas para a desigualdade de renda no Brasil rural. Economia Aplicada, São Paulo, v. 12, n. 3, p. 265-393, jul./set. 2008.

. e HOFFMANN, R. Educação, concentração fundiária e desigualdade de rendimentos no meio rural brasileiro. Revista e Economia e Sociologia Rural, Piracicaba, 2009.

MAIA GOMES, G. Aposentados e funcionários públicos: a economia sem produção: velhas secas em novos sertões: continuidade e mudanças na economia do semiárido e dos serrados nordestinos. Brasília, DF: IPEA, 2001, p. 145-175.

MARIANO, J. L. e LOPES, T. S. Efeitos da previdência social sobre a desigualdade e a pobreza rural no Nordeste: uma análise da decomposição do índice de Gini. Revista de Economia do Nordeste. Fortaleza, v. 40, n. 1, jan./mar. 2009.

MATSHE, I. e YOUNG, T. Off-farm labor allocation decisions in small-scale rural households in Zimbabwe. Agricultural Economics, p. 175-186, v. 30, n. 3, 2004. 
MESQUITA, S. P., SAMPAIO, L. M. B., RAMALHO, H. M. B. e ARAÚJO JÚNIO, I. T. A oferta de trabalho não agrícola no meio rural: evidências para Nordeste e o Brasil. Revista Economiaa, Brasília (DF), v. 11, n. 4, p. 103131, dez. 2010.

REARDON, T., BERDEGUE, J., BARRETT, C. B. e STAMOULIS, K. Household Income Diversification into Rural Nonfarm Activities. In: HAGGBLADE, HAZELL e REARDON (Eds.). Transforming the Rural Nonfarm Economy. Baltimore, MD: Johns Hopkins University Press, forthcoming, 2006.

ROCHA, S. O programa Bolsa Família: evolução e efeitos sobre a pobreza. Economia e Sociedade, Campinas, v. 20, n. 1(41), p. 113-139, 2011.

SANTOS, G. S., FONTES, R. M. O., BASTOS, P. M. A., e LIMA, J. E. Mercado de trabalho e rendimento no meio rural brasileiro. Economia Aplicada, v. 14, n. 3, p. 355-379, 2010.

SCHNEIDER, S. A pluriatividade no meio rural brasileiro: características e perspectivas para investigação. In: GRAMMONT, H. C. e MARTINEZ VALE, L. (Org.). La pluriactividad en el campo latinoamericano. Quito. Ed. Flacso -séria FORO, v. 1, p. 132-161, 2009.
SETSOFFIA, E. D., SARPONG, D. B. e KWADZO, B. T. M. Determinants of direct marketing by agro SMEs' in the greater accra region, Ghana. Asian journal agricultural extension, Economics \& Sociology, v. 2, n. 2, 2013.

SOARES, F. V., RIBAS, R. P. e OSÓRIO, R. G. Avaliando o impacto do Programa Bolsa Família: uma comparação com programas de transferência condicionada de renda de outros países. Brasília, DF/Centro Internacional da Pobreza: BNDES, 2007.

TAVARES, P. A. et al. Uma avaliação do Programa Bolsa Família: focalização e impacto na distribuição de renda e pobreza. Ribeirão Preto: Mimeo, 2008.

TEIXEIRA, C. G. Análise do impacto do Programa Bolsa Família na oferta de trabalho dos homens e mulheres. In: SILVA, F. S. (Org.). I Prêmio e I Mostra Nacional de estudos sobre o Programa Bolsa Família. Brasília/DF: Ministério do Desenvolvimento Social, 2008. Disponível em: <http://www.ipc-undp.org>. Acesso em: 15 abr. 2012.

WODJAO, T. B. A Double-hurdle model of computer and internet use in american households. Western Michigan University, 2007. 
